\title{
A lesson from COVID-19 on inaccessibility of web-based information for disabled populations worldwide
}

\author{
Amiel A. Dror ${ }^{1,2}$, Eli Layous ${ }^{1,2}$, Matti Mizrachi, ${ }^{1,2}$, Amani Daoud ${ }^{1,2}$, Netanel \\ Eisenbach ${ }^{1,2}$, Nicole G. Morozov ${ }^{4}$, Samer Srouji ${ }^{2,3}$, Karen B. Avraham ${ }^{5,}$, Eyal \\ Sela1,2,*
}

${ }^{1}$ Department of Otolaryngology, Head and Neck Surgery, Galilee Medical Center, Nahariya, Israel; ${ }^{2}$ Azrieli Faculty of Medicine, Bar-Ilan University, Safed, Israel; ${ }^{3}$ Oral and Maxillofacial Department, Galilee Medical Center, Nahariya, Israel; ${ }^{4}$ Sackler School of Medicine, Tel Aviv University, Tel Aviv, Israel; ${ }^{5}$ Department of Human Molecular Genetics and Biochemistry, Sackler Faculty of Medicine and Sagol School of Neuroscience, Tel Aviv University, Tel Aviv, Israel

${ }^{*}$ Equal authors

Corresponding author: Amiel A. Dror, Department of Otolaryngology, Head and Neck Surgery, Galilee Medical Center, Nahariya, Israel; Tel: +972544530534; email: amieldror@gmail.com 


\begin{abstract}
Many government websites and mobile content are inaccessible for people with vision, hearing, and cognitive disabilities. The COVID-19 pandemic highlighted these disparities when health authority website information, critical in providing resources for curbing the spread of the virus, remained inaccessible for disabled populations. The Web Content Accessibility Guidelines provide comparatively universally accepted guidelines for website accessibility. We utilized these parameters to examine the number of countries with or without accessible health authority websites. The resulting data indicate a dearth of countries with websites accessible for persons with disabilities. Methods of information dissemination must take into consideration individuals with disabilities, particularly in times of global health crises.
\end{abstract}

\title{
Introduction
}

The COVID-19 pandemic is challenging the boundaries of not only social behaviors and cultural institutions, but also the rapid and accurate dissemination of information. The containment of this epidemic has required stringent adherence to interpersonal behavioral modifications, which are often developed and transmitted by national health authorities. However, national health authority websites may lack website accommodations for people with vision, hearing, physical, or cognitive impairments. Minimizing the information gap between able-bodied and disabled populations is imperative for achieving global engagement in containing not only COVID-19, but also future pandemics. We sought to determine what percentage of national health authority websites are fully accessible to people with disabilities according to Web Content Accessibility (WCAG 2.1) guidelines (W3C, 2020a) benchmarks. Our research demonstrates that only a small percentage of government health websites are fully accessible for people with disabilities.

According to the World Health Organization (WHO), an estimated 2.2 billion people suffer from vision impairment or blindness (WHO Blindness and vision impairment, 2019), while 466 million people have a disabling hearing loss (WHO Deafness and hearing loss, 2020). Individuals with temporary or permanent motor or cognitive disabilities also require accessibility modifications for proper interaction with websites. Inconsistent heading level and font size or color contrast of elements in webpages harbor barriers for proper interaction by visually impaired people. Likewise, alternative textual descriptions of visual elements on a page are essential for contextual understanding, in addition to proper interaction with text-to-speech engines. Lack of video content subtitles or transcripts present barriers to the hearing impaired. Compatibility with keyboard navigation, including skip linking in the backend of a website, is crucial to accommodate web navigation for people with motor dysfunction who interact with a single finger or with other motor gestures.

\section{Materials and methods}

The Web Accessibility Initiative (WAI), launched and endorsed by the World Wide Web Consortium (W3C) (W3C, 2020b), established a set of guidelines according to four accessibility principles: whether the website is Perceivable, Operable, Understandable, and Robust. Each WCAG 2.1 principle has a set of testable criteria with a total number of 78 testable success criteria. Each success criteria is assigned to one of three conformance levels: A (lowest), AA (intermediate), and AAA (highest). The adherence to higher levels of conformance has been shown to improve accessibility for both disabled and nondisabled users (Loiacono and Djamasbi, 2013; Ruth-Janneck, 2011; Schmutz et al., 2016). A panoply of web accessibility evaluation plug-ins was developed under open-source license for the systematic evaluation of website accessibility against the WCAG 2.1 criteria (W3C, 2020a). A list of available tools is presented by the $\mathrm{W} 3 \mathrm{C}$ website without an official recommendation for usage of one tool above 
another (W3C, 2020c). These automated tools aim to complement the cardinal manual check of a website during the development process and throughout routine website updates to ensure maximal adherence WCAG guidelines (Petrie and Bevan, 2009). A comprehensive comparison between eight widely used accessibility evaluation tools highlights the strengths and weaknesses of each tool and recommends using more than one tool for optimal coverage of success criteria (Frazão and Duarte, 2020). Hence, to test the accessibility of COVID-19 information disseminated through health authority websites, we utilized two independent accessibility evaluation engine including WAVE chrome extension (wave.webaim.org) and Accessibility Insights (accessibilityinsights.io), both of which have been described and utilized in previous literature (Acosta-Vargas et al., 2020; Frazão and Duarte, 2020). The WAVE tool analyzes 180 checks according to two conformances level (152 level A; 28 level $A A)$; whereas the Accessibility Insights tool analyzes 64 checks according to three conformances level (55 level A; 7 level AA; and 2 level AAA) (Frazão and Duarte, 2020). It must be noted that the weight of each error (e.g. minor, moderate, critical) is defined by the tool developer and thus may result in different impacts on the overall accessibility rank of the page results.

Due to the rapid growth of COVID-19 information and the frequent updates of health authorities' websites, which may influence the accessibility score at a given time point, the degree of accessibility of each website was evaluated at three different time points and the presented data refer to the following three consecutive days (5-7 April, 2020). The calculated number of errors of each health authority homepage augments the average number of errors in each test separately (WAVE and Accessibility Insights), with removal of redundant errors represented in both tests.

In addition to accessibility assessments, we tested each website for mobile usability in concordance to Google webmaster developer tools (developers.google.com). In this regard, previous studies have demonstrated that mobile-friendliness of a given website contributes not only to end user usability, but also for website visibility on search engine results (Schubert, 2016).

The list of health authorities' websites of 189 countries were drawn from The Geneva Foundation for Medical Education and Research (GFMER, 2020) (Supplementary Table 1). Prior to accessibility evaluation, a manual check of each website on the list yielded 174 health authority websites. Websites of 15 countries were excluded due to an inability to load the site on the test server or when the official health authority homepage appeared as a social media page. This was a crosssectional study concentrating on the accessibility of health authorities' websites' homepages (unit of analysis) providing health information and recommended public protective measures against COVID19.

\section{Results}

Only $4.7 \%$ of the countries examined had fully implemented the WAI accessibility guidelines: Italy, the Netherlands, Norway, Japan, Poland, South Korea, the United Kingdom, and the United States (Figure 1). In contrast, sites from the majority of countries continue to have accessibility errors that present significant barriers to people with disabilities around the world. Distribution of reported errors across all 174 tested health authorities' homepages, according to WCAG conformance levels, reveals that $89 \%$ violate Level A criteria, while $11 \%$ of countries contain errors that violate higher levels of success criteria (AA and AAA). Inspection of the numbers of errors on all tested pages grouped by WCAG principles indicate that the most impacted principles are robustness (39\%) and perceptibility (32\%), as compared to operability (19\%) and understandability (10\%). While both error number, conformance, and principle distribution may be altered according to the selected assessment tools, the data collected signifies the insufficient implementation of WCAG guidelines in the majority of health authority websites, rendering accessibility barriers to millions of people. 


\section{(7) ACCESSIBILITY ERRORS ACCORDING TO WCAG 2.1 CRITERIA}

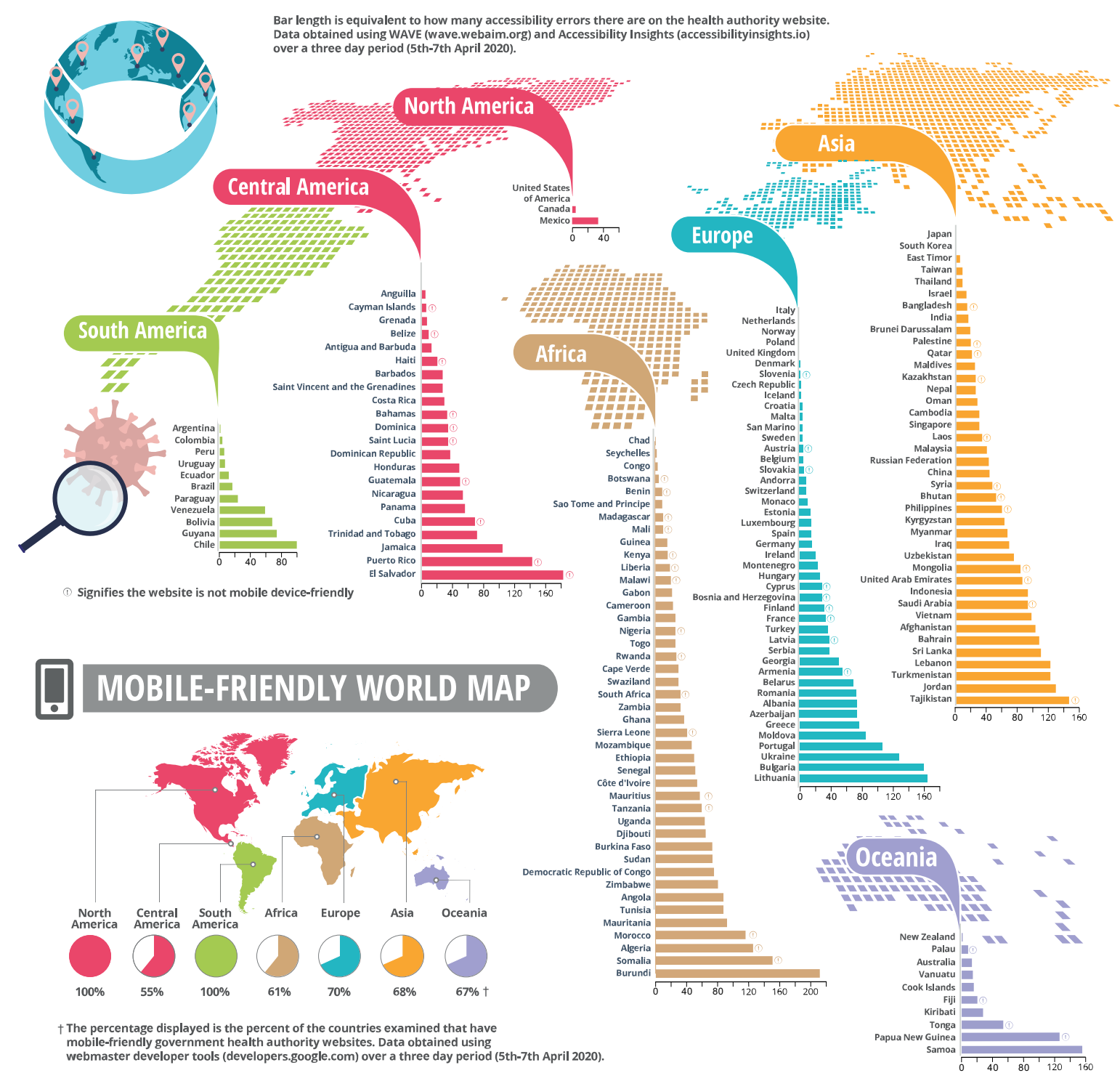

Figure 1. Health authority websites of 174 countries worldwide, demonstrating accessibility errors and mobile friendly maps. A. The calculated number of errors of each health authority website augment the number of error results in each accessibility evaluation tool separately (WAVE and Accessibility Insights), following obliteration of redundant errors that are represented in both tests. B. Mobile computability check according to the Google web developer tool with either pass of fail results. All tests were performed on three consecutive days (5-7 April 2020). 


\section{Discussion}

Reducing transmission of SARS-CoV-2 depends on tight adherence of the public to simple but challenging modifications in social and public behavior (Chu et al., 2020). Digital media provide numerous platforms to distribute essential information to the public through websites, social media, and instant messaging applications (Jung and Shin, 2020).

Due to the diversity of reporting sources and the harmful consequences of disinformation (Islam et al., 2020), governments often encourage the public to check local health authority websites frequently for regular updates. This demand requires the information on official websites to be accessible to as many citizens as possible. Unfortunately, individuals with the greatest need for timely and precise data may have the most difficulty accessing governmental material (West and Miller, 2006). Providing consistently high-quality government productions could also lead to a greater utilization of the Internet by persons with disabilities. Enhancing accessibility to governmentsponsored resources could lead not only to immediate population benefits but could also promote the position of disabled people in the digital sphere through increased communication, global engagement, and visibility.

Despite remarkable technological advancements in recent history, for people with visual, hearing, motor and cognitive disabilities, a seemingly simple website interaction can present a daunting challenge. Although internet access is still unavailable to approximately one-third of the world's population, the needs of all existing users must be accommodated to ensure equal benefits and access to essential health information. The growth and expansion of the Internet must therefore be accompanied by an equal development of sophisticated accessibility technologies, which would expand the usability of the web to individuals with disabilities. Without underestimating the importance of accessibility implementation during normal times, the current COVID-19 pandemic now highlights just how important unhindered access to government websites is during a global health crisis.

\section{Competing interests}

The authors have no other competing interests to declare.

\section{Author ORCIDs}

Amiel A. Dror, https://orcid.org/0000-0002-7178-6771

Netanel Eisenbach, https://orcid.org/0000-0003-4513-3766

Samer Srouji, https://orcid.org/0000-0001-8837-2391

Karen B. Avraham, https://orcid.org/0000-0002-4913-251X 


\section{References}

Acosta-Vargas P, González M, Luján-Mora S. 2020. Dataset for evaluating the accessibility of the websites of selected Latin American universities. Data in Brief 28:105013. https://doi.org/10.1016/j.dib.2019.105013

Chu DK, Akl EA, Duda S, Solo K, Yaacoub S, Schünemann HJ. 2020. Physical distancing, face masks, and eye protection to prevent person-to-person transmission of SARS-CoV-2 and COVID-19: a systematic review and meta-analysis. The Lancet 395:1973-1987.

DOI:https://doi.org/10.1016/S0140-6736(20)31142-9

Frazão T, Duarte C. 2020. Comparing accessibility evaluation plug-ins. Association for Computing Machinery. 20:1-11. https://doi.org/10.1145/3371300.3383346

Geneva Foundation for Medical Education and Research. 2020. Ministries of health worldwide. https://www.gfmer.ch/Medical_search/Ministry_health.html [Accessed August 14, 2020].

Islam MS, Sarkar T, Khan SH, Mostofa Kamal A-H, Hasan SMM, Kabir A, Yeasmin D, Islam MA, Amin Chowdhury KI, Anwar KS, Chughtai AA, Seale H. 2020. COVID-19-related infodemic and its impact on public health: A global social media analysis. The American Journal of Tropical Medicine and Hygiene DOI:https://doi.org/10.4269/ajtmh.20-0812

Jung J-H, Shin J-I. 2020. Big data analysis of media reports related to COVID-19. International Journal of Environmental Reseach and Public Health 17: 5688.

DOI:https://doi.org/10.3390/ijerph17165688

Loiacono ET, Djamasbi S. 2013. Corporate website accessibility: does legislation matter? Universal Access in the Information Society 12:115-124. DOI: https://doi.org/10.1007/s10209-011-0269-1

Petrie H, Bevan N. 2009. The evaluation of accessibility, usability, and user experience. The Universal Access Handbook. C Stepanidis (ed), CRC Press. pp 1-30. DOI:10.1201/9781420064995-c20

Ruth-Janneck D. (2011) Experienced Barriers in Web Applications and Their Comparison to the WCAG Guidelines. In: Holzinger A., Simonic KM. (eds) Information Quality in e-Health. USAB 2011. Lecture Notes in Computer Science, vol 7058. Springer, Berlin, Heidelberg. https://doi.org/10.1007/978-3-642-25364-5_21

Schmutz S, Sonderegger A, Sauer J. 2016. implementing recommendations from web accessibility guidelines: Would they also provide benefits to nondisabled users. Human Factors 58:611-629. https://doi.org/10.1177/0018720816640962

Schubert D. 2016. Influence of mobile-friendly design to search results on Google search. Procedia Social and Behavioral Sciences 220:424-433. DOI:https://doi.org/10.1016/j.sbspro.2016.05.517 W3C. 2020a. Web Content Accessibility Guidelines (WCAG) 2.1. https://www.w3.org/TR/WCAG21/ [Accessed August 14, 2020].

W3C. 2020b. Web Content Accessibility Guidelines (WCAG) Overview. https://www.w3.org/WAl/standards-guidelines/wcag/ [Accessed August 14, 2020].

W3C. 2020c. Selecting Web Accessibility Evaluation Tools. https://www.w3.org/WAl/testevaluate/tools/selecting/ [Accessed August 14, 2020].

West DM, Miller EA. 2006. The digital divide in public e-health: barriers to accessibility and privacy in state health department websites. Journal of Health Care Poor for the Poor and Underserved 17:652-667. DOI:https://doi.org/10.1353/hpu.2006.0115

WHO Blindness and vision impairment. 2019. Blindness and vision impairment. https://www.who.int/news-room/fact-sheets/detail/blindness-and-visual-impairment [Accessed August 14, 2020]

WHO Deafness and hearing loss. 2020. Deafness and hearing loss. https://www.who.int/newsroom/fact-sheets/detail/deafness-and-hearing-loss [Accessed August 14, 2020] 